\title{
Sleepless in Chicago: Tracking the Effects of Adolescent Sleep Loss During the Middle School Years
}

\author{
Katia Fredriksen, Jean Rhodes, Ranjini Reddy, and Niobe Way
}

\begin{abstract}
The influence of the sleep patterns of 2,259 students, aged 11 to 14 years, on trajectories of depressive symptoms, self-esteem, and grades was longitudinally examined using latent growth cross-domain models. Consistent with previous research, sleep decreased over time. Students who obtained less sleep in sixth grade exhibited lower initial self-esteem and grades and higher initial levels of depressive symptoms. Similarly, students who obtained less sleep over time reported heightened levels of depressive symptoms and decreased self-esteem. Sex of the student played a strong role as a predictor of hours of sleep, self-esteem, and grades. This study underscores the role of sleep in predicting adolescents' psychosocial outcomes and highlights the importance of using idiographic methodologies in the study of developmental processes.
\end{abstract}

A host of social, academic, and biological changes conspires to diminish the amount and quality of sleep that young adolescents obtain over time. Adolescents go to bed later, get up earlier, and feel increasingly sleepy during the day (Andrade \& Menna-Barreto, 2002; Carskadon, 1990b; Laberge et al., 2001). Later bedtimes combined with earlier school start times often mean that, as adolescents mature, they obtain much less sleep than they did in preadolescence (Carskadon, Wolfson, Acebo, Tzischinsky, \& Seifer, 1998; Dahl \& Lewin, 2002; Wolfson \& Carskadon, 1998). This can lead to chronic insufficient sleep with additive effects that can have serious negative implications (Carskadon \& Dement, 1987; Dahl \& Lewin, 2002). In this study, we focused on the role of sleep in predicting academic and psychosocial outcomes. To accommodate for individual variation and developmental

Katia Fredriksen, Jean Rhodes, and Ranjini Reddy, Department of Psychology, University of Massachusetts Boston; Niobe Way, Department of Applied Psychology, New York University.

We gratefully acknowledge the contribution of the researchers at the Illinois Center for Prevention Research and of Robert D. Felner, who served as the principal investigator of the Alliance of Illinois Middle Schools network evaluation, and Peter Mulhall who coordinated the project. The evaluation was funded by a Carnegie Foundation grant to the University of Illinois. A grant from the Spencer Foundation to Jean Rhodes and Niobe Way permitted the analyses in this study. We would also like to thank Aline Sayers for her assistance with the analyses and the anonymous reviewers for their insightful feedback and suggestions.

Correspondence concerning this article should be addressed to Jean Rhodes, Department of Psychology, University of Massachusetts Boston, 100 Morrissey Boulevard, Boston, MA 02125-3393. Electronic mail may be sent to jean.rhodes@umb.edu. changes in sleep, we incorporated modeling techniques that assessed the effects of participant sleep on the outcome variables as they evolved from the beginning of sixth grade through the end of eighth grade. Finally, we examined the role of sex as a predictor of sleep patterns and effects.

\section{Background}

As many parents will attest, adolescents often burn the candle at both ends - they stay up late finishing homework or corresponding with friends and then struggle out of bed at dawn to beat the first school bell. Although they log fewer hours of sleep than elementary school age children, laboratory data indicate that adolescents may, in fact, need more (Dahl \& Lewin, 2002). In a longitudinal sleep lab study, Carskadon, Harvey, Duke, Anders, and Dement (1980) found that sleep quantity remained the same at just over $9 \mathrm{hr}$ throughout adolescence, indicating that, ideally, adolescents would obtain a consistently high level of sleep as they matured. By midadolescence, adolescents tended to be more tired during the day, and older adolescents became tired in the daytime even when they received as much sleep as younger adolescents (Carskadon, 1990a). Dahl and Carskadon (1995) maintained that prepubescent children sleep efficiently, engaging in deep sleep at night that leaves them alert during the day. Although children begin to sleep more lightly as they get older, marked declines in deep sleep make it difficult for adolescents to obtain the amount necessary to remain fully alert during the day (Dahl \& Lewin, 2002). Dahl and Caskadon

(C) 2004 by the Society for Research in Child Development, Inc. All rights reserved. 0009-3920/2004/7501-0005 
(1995) concluded that "if anything, adolescents require more sleep ... [but] they frequently obtain much less" (p. 19).

A number of biological and contextual variables play a pivotal role in adolescent sleep patterns (Carskadon, 1999; Carskadon, Acebo, Richardson, Tate, \& Seifer, 1997; Carskadon et al., 1998; Dornbusch, 2002). For instance, pubertal status and morningness or eveningness (i.e., being a morning person or an evening person) are related. Eveningtype adolescents spend less time sleeping on school nights than morning types and are less able to adjust their sleep schedules to earlier school start times (Carskadon, Vieira, \& Acebo, 1993; Giannotti, Cortesi, Sebastiani, \& Ottaviano, 2002). In addition, researchers have shown that adolescents may actually adopt a circadian period of more than $24 \mathrm{hr}$, which contributes to adolescent phase delays in sleep (Carskadon, Labyak, Acebo, \& Seifer, 1999). A typical cycle of sleep deficiency and daytime sleepiness occurs when adolescents indulge in catch-up sleep on the weekends and holidays. This leads to an even later circadian schedule, resulting in an even more imbalanced sleep-wake schedule when they return to school and are required to awaken early again (Dahl \& Carskadon, 1995; Dahl \& Lewin, 2002). Although this phase delay and sleep loss begins in childhood (Sadeh, Raviv, \& Gruber, 2000), it becomes increasingly marked as adolescence progresses (Park et al., 2001; Park, Matsumoto, Seo, Kang, \& Nagashima, 2002).

At the same time, part-time jobs and a growing array of extracurricular and social activities increasingly compete with sleep time (Carskadon, 1999; Dahl \& Carskadon, 1995; Dahl \& Lewin, 2002; Wolfson, 2002), especially in conjunction with a decreased likelihood that parents are setting bedtimes (Carskadon, 1999, 2002). Changes also occur at school, where tougher academic demands necessitate that more time be devoted to homework. In addition, greater computer use for school assignments often includes nonacademic use of the Internet for games and socializing. In fact, in recent years there has been a growing concern about Internet addiction (Young, 1998), to which adolescents are particularly susceptible (Hall \& Parsons, 2001; Kandell, 1998).

School days tend to begin progressively earlier as students move from elementary to middle school and from middle school to high school (Carskadon, 2002), further exacerbating the sleep loss. School start times are difficult to change because teachers, parents, students, and members of the community at large are all affected by them (Wahlstrom, 1999,
2002). Nonetheless, earlier start times are associated with significant sleep deprivation, daytime fatigue, irregular sleep schedules, and the tendency to fall asleep at school, as adolescents with earlier school start times go to bed just as late as adolescents with later school start times (Carskadon et al., 1998; Dahl \& Lewin, 2002; Giannotti \& Cortesi, 2002).

This clash between bodily rhythms and contextual factors can have a wide variety of negative consequences. Most research on this topic has focused on the academic realm, demonstrating impairments in sleep-deprived students (Carskadon, 1990b). Although focused on an older age range, Wolfson and Carkasdon (1998) found that high school students who reported that they were struggling at or failing school went to bed an average of $40 \mathrm{~min}$ later than students with better grades. Insufficient sleep has been shown to compromise learning, memory, attention, and abstract thinking skills (Carskadon, 1999; Randazzo, Muehlbach, Schweitzer, \& Walsh, 1998; Sadeh et al., 2000). In addition, insufficient sleep results in an increased risk for school absences because of physical illness, falling asleep in school, oversleeping in the morning, fatigue, and irritability, all of which are detrimental to academic outcomes (Dahl \& Carskadon, 1995; Roberts, Roberts, \& Chen, 2001).

Although subject to less research, another area of concern involves the psychosocial consequences of sleep deprivation. Depression causes sleep loss, yet researchers have speculated that this may in fact be a bidirectional causal pathway (Allgoewer, Wardle, \& Steptoe, 2001; Dahl \& Lewin, 2002). Carskadon (1999) found that sleep deprivation led to depressed mood, and similarly, Roberts et al. (2001) noted that disturbed mood, suicide ideation, and unhappiness were among the strongest correlates of insomnia. Other researchers have also linked suicide ideation with tiredness (Choquet, Kovess, \& Poutignat, 1993). In addition, Wolfson and Carskadon (1998) found that high school students who obtained less sleep on school nights or experienced greater discrepancies between school night and weekend night bedtimes, or both, were more likely to suffer from depressed mood. The researchers concluded that "adolescent moodiness may be in part a repercussion of insufficient sleep" (p. 885). This moodiness could influence coping skills and relationships with peers and adults (Carskadon, 1990b). Insufficient sleep also has an impact on affect. Dahl and Lewin (2002) suggested that sleep loss lessens adolescents' control over their mood. Indeed, Leotta, Carskadon, Acebo, Seifer, and Quinn (1997) found that sleep-deprived 
participants aged 10 to 15 years reported significantly higher levels of anger, sadness, and fear toward negative slides.

\section{Current Study}

Previous findings suggest that for a variety of reasons adolescents obtain steadily decreasing levels of sleep and that this sleep deprivation is associated with negative academic and psychosocial outcomes. Nonetheless, many previous studies of this important topic have been limited by small samples, crosssectional designs, and multivariate methods that take a static view of a period that is in constant flux. We were able to address these limitations by using cross-domain latent growth modeling to analyze date from a large $(N=2,259)$ sample of adolescents as they progressed through 3 years of middle school. Cross-domain latent growth modeling permitted us to examine the effects of change in sleep on change in academic and psychosocial outcome variables over time.

We hypothesized that there would be a precipitous drop in sleep as participants progressed from sixth to eighth grade. We expected that insufficient sleep would negatively affect both academic and psychosocial variables such as depressive symptoms and self-esteem. In addition to looking at trends in the overall sample, we examined student background characteristics as predictors of sleep loss. Few past studies have focused on such influences as sex of participants on sleep patterns. Nonetheless, Giannotti and Cortesi (2002) found that girls were significantly more likely than boys to report poor sleep quality. In addition, Lee, McEnany, and Weekes (1999) found that boys woke up significantly later on weekdays than girls, in part because they spend less time grooming in the morning. Thus, although we anticipated that bedtimes would drift later for both girls and boys throughout adolescence, we expected that boys would obtain more sleep than girls. Research has shown that girls are more vulnerable to drops in self-esteem and rises in depression, and if sleep does indeed affect these variables, this could contribute to a greater understanding of the sex differences in adolescent psychosocial well-being (Hankin \& Abramson, 2001; Kling, Hyde, Showers, \& Buswell, 1999). We also considered socioeconomic factors that might be predictive of decreased sleep. For example, because lower income, minority youth are more likely to live in crowded quarters and noisy neighborhoods and to shoulder jobs and household responsibilities (Furstenberg, Cook, Eccles, Elder, \& Sameroff, 1999), we anticipated that they would obtain fewer hours of sleep than higher socioeconomic status (SES), White students.

\section{Method}

Data were drawn from a larger, ongoing longitudinal assessment of young adolescents in middle school. The 23 participating schools were all members of the Association of Illinois Middle-Level Schools, serving students from geographically, socioeconomically, and racially diverse backgrounds. Administrators in the schools agreed to participate in the study as part of a statewide school assessment plan, which included both student and staff surveys during the spring of each academic year. The current study focused on the self-report data of students who completed the survey during three consecutive years, starting in sixth grade, from 1995 to 1997.

Of the original 2,860 students, approximately 9\% were dropped from the study because of missing demographic information. Although listwise deletion is the most commonly used procedure for handling missing data, we chose not to use this procedure as it would have necessitated eliminating an additional 963 students from our analyses (dropping our sample size of 2,259 to 1,296). Instead, we estimated the parameters in the presence of missing data, using the full information maximum likelihood (FIML) procedure available in LISREL 8.51 (Jöreskog \& Sörbom, 2001). The FIML procedure in LISREL uses the expectation maximization (EM) algorithm to obtain estimates of the population means and covariances; these estimates are then used to obtain starting values for the maximum likelihood procedure (duToit \& duToit, 2001). The advantage of this procedure is that it uses all the information of the observed data.

\section{Participants}

The sample consisted of 2,259 students who began sixth grade in 1995 and completed eighth grade in 1997. Participants ranged in age from 11 to 14 years. Half of the sample was male $(50.4 \%)$, and the majority identified themselves as European Americans (82.2\%). The remaining participants were Hispanic (6.9\%), African American (5.4\%), Asian American (3.4\%), multiracial $(1.8 \%)$, and Native American $(0.3 \%)$. Eligibility to receive free or reduced price lunches was interpreted as an indication of low-income status; approximately $23.4 \%$ fell into this category at the beginning of middle school. Slightly more than one third of the students reported that their mothers had completed college (36.9\%). 
The majority of the students (66\%) reported living in two-parent households at the end of middle school (73\% lived in two-parent households at the beginning of the study). The adolescents were drawn from 23 Midwestern schools that housed Grades 6 through 8 and had enrollments ranging from 203 to 1,243 students. The percentage of White students ranged from $1.6 \%$ to $100 \%$, and the percentage of low-income students ranged from $0.1 \%$ to $95.5 \%$.

\section{Measures}

Teachers administered the surveys to students in their homeroom classrooms. Instructions and individual items were read aloud by the teachers while the students read along silently. The surveys were typically completed in 2 class hours, usually over a 2-day period. Before seeking parental consent, the surveys and consent forms were approved by school-based parent advisory groups. Fewer than $10 \%$ of parents refused to provide consent for their children to participate.

Sleep. To assess the amount of sleep participants received on school nights, the following question was included in the survey: "How many hours of sleep do you get on a typical school night?" Participants selected from the following responses: "5 hours or less," "6 hours," "7 hours," "8 hours," and "9 or more hours."

Academic outcomes. Academic outcomes were also self-reported in response to the following question: "What kinds of grades did you earn in school last year?" Participants responded "Mostly A's and B's," "Mostly B's," "Mostly B's and C's,", "Mostly C's," or "Mostly D's and below."

Depressive symptoms. Depressive symptoms were indexed with a shortened version of the Children's Depressive Inventory (CDI; Kovacs, 1980), the most frequently used scale to assess depressive symptomatology in children (Tvenge \& Nolen-Hoeksema, 2002). The 16-item self-report scale measures cognitive, affective, and behavioral symptoms of depression. For each item, students selected one of three statements (increasing in symptom severity) that best described their feelings and ideas during the previous 2 weeks. For example, they chose among statements such as "I am sad once in a while," "I am sad much of the time," and "I am sad all the time." Item choices are assigned a numerical value from 0 to 2 , and the scale yields a single aggregate measure of depressive symtomolotogy reflecting an underlying unidimensional construct. Lower scores index lower levels of reported depressive symptoms. The CDI has shown good test-retest reliability, internal consistency, and construct validity, especially in nonclinical populations (Sitarenios \& Kovacs, 1999). Scores on the CDI are moderately correlated with psychiatrists' ratings of children's depression (Kazdin, 1989). Although the CDI does not appear to make a clear distinction between symptoms of depression and symptoms of anxiety, these symptoms tend to be highly comorbid in children and adolescents, whereas pure depression is relatively rare (Kovacs \& Devlin, 1998; Saylor, Finch, Spirito, \& Bennett, 1984). Internal consistency for the three years was $\alpha=.85, \alpha=.84$, and $\alpha=.89$, respectively.

Self-esteem. Self-esteem was indexed by the global self-esteem subscale of the Self-Esteem Questionnaire (SEQ; DuBois, Felner, Brand, Phillips, \& Lease, 1996). The scale consists of eight items that assess overall perceptions of self-worth. For example, students rated statements such as "I like being the way I am," " I am happy with myself as a person," and "I am as good a person as I want to be" on a 4point scale (from strongly disagree to strongly agree) with higher ratings indicating higher levels of selfesteem. Prior research has yielded strong support for the psychometric properties of the SEQ (DuBois et al. 1996; DuBois \& Felner, 1991), including satisfactory levels of internal consistency and test-retest reliability, as well as factor analytic findings consistent with the subscales across demographically diverse young adolescents (see Dubois, Bull, Sherman, \& Roberts, 1998; Dubois, Felner, Brand, \& George, 1999). Internal consistency of the subscale was $\alpha=.82$ (Time 1), $\alpha=.84$ (Time 2), and $\alpha=.84$ (Time 3).

Demographic characteristics. Single items were used to assess students' grade level, sex (female $=1$ ), race or ethnicity (White $=1$, non-White $=0$ ), whether they received free or reduced price lunch at school (free or reduced price lunch $=1$ ), mother's educational level (college graduate $=1$ ), and with whom they lived in the last year.

\section{Results}

\section{Descriptive Analysis}

In this and the following sections we provide descriptive analyses of the sleep and outcome data followed by findings derived from the cross-domain latent growth modeling. Table 1 presents the means, standard deviations, and univariate skewness and kurtosis coefficients for sleep and the outcome variables. The variables were normally distributed with no significant skewness or kurtosis; skewness coefficients ranged from .52 to 1.54 , whereas kurtosis 
Table 1

Means, Standard Deviations, Univariate Skewness and Kurtosis for Sleep and the Outcome Measures

\begin{tabular}{lcccr}
\hline Variables & Mean & SD & Skewness & Kurtosis \\
\hline Time 1: Hours of Sleep & 3.88 & 1.13 & -0.99 & 0.33 \\
Time 2: Hours of Sleep & 3.67 & 1.14 & -0.8 & -0.05 \\
Time 3: Hours of Sleep & 3.37 & 1.16 & -0.52 & -0.51 \\
Time 1: Depressive & 1.35 & 0.33 & 1.34 & 1.95 \\
Time 2: Depressive & 1.34 & 0.34 & 1.47 & 2.22 \\
Time 3: Depressive & 1.39 & 0.39 & 1.54 & 2.65 \\
Time 1: Self-Esteem & 3.14 & 0.58 & -0.56 & 0.24 \\
Time 2: Self-Esteem & 3.04 & 0.6 & -0.55 & 0.39 \\
Time 3: Self-Esteem & 2.98 & 0.6 & -0.52 & 0.52 \\
Time 1: Grades & 4.31 & 1.07 & -1.36 & 0.77 \\
Time 2: Grades & 4.15 & 1.19 & -1.09 & -0.06 \\
Time 3: Grades & 4.01 & 1.25 & -0.93 & -0.39 \\
\hline
\end{tabular}

Note. For Hours of Sleep, Mean $=3.88$ for Time 1: Hours of Sleep $=$ nearly 8 hours of sleep; Mean $=3.67$ for Time $2=$ just over 7 and a half hours of sleep; Mean $=3.37$ for Time $3=$ between 7 and 7 and a half hours of sleep. For Depressive Symptoms, item choices on the CDI were assigned numerical values of $0-2$, with lower scores indexing lower levels of reported depressive symptoms. For Self-Esteem, the SEQ used a four-point scale, with higher ratings indicating higher levels of self-esteem. For Grades, participants selected 5= "Mostly A's and B's," $4=$ "Mostly B's," $3=$ "Mostly B's and C's," 2 = "Mostly C's,", or $1=$ "Mostly D's and below," such that a lower mean indicates lower grades.

ranged from -0.51 to 2.65 . As predicted, overall observed means showed declines in hours of sleep, self-esteem, and self-reported grades, and a rise in depressive symptoms. The pattern of associations between the predictors and the outcomes indicated significant correlations among the variables in the expected direction (see Table 2). Hours of sleep in eighth grade were negatively correlated with depressive symptoms in eighth grade and positively correlated with self-esteem in eighth grade, such that students obtaining less sleep were more likely to report depressive symptoms and low self-esteem. There was a positive correlation between eighthgrade sleep and self-reported grades, such that students obtaining more sleep reported slightly higher grades.

\section{Analytical Strategy}

Cross-domain latent growth modeling was the primary method used to test the hypotheses of this study. This method involved examining withinperson and between-person growth curves. The first step in this process involved using latent growth modeling strategies to define the Level 1, withinperson individual growth curves. The graphical representation of the analyzed model can be seen in Figure 1. Observed data (i.e., covariances among measures at each time point) were mapped onto a measurement model. The measurement model represented individual growth in each construct and was defined by two growth parameters, the intercept and the slope.

An important step in defining the growth parameters, the intercept and the slope, was the selection of a suitable statistical model to represent change over time. With three waves of data, a linear trajectory of growth was selected for the current model. Observations were taken once each academic year from sixth

Table 2

Zero-Order Correlations for Sleep, the Outcome Measures, and the Demographic Variables

\begin{tabular}{|c|c|c|c|c|c|c|c|c|c|c|c|c|c|c|c|}
\hline Variable & 1 & 2 & 3 & 4 & 5 & 6 & 7 & 8 & 9 & 10 & 11 & 12 & 13 & 14 & 15 \\
\hline \multicolumn{16}{|l|}{ 1. Time 1: hours of sleep } \\
\hline 2. Time 2: hours of sleep & .32 & & & & & & & & & & & & & & \\
\hline 3. Time 3: hours of sleep & .26 & .34 & & & & & & & & & & & & & \\
\hline 4. Time 1: depressive symptoms & -.25 & -.21 & -.19 & & & & & & & & & & & & \\
\hline 5. Time 2: depressive symptoms & -.21 & -.34 & -.23 & .50 & & & & & & & & & & & \\
\hline 6. Time 3: depressive symptoms & -.18 & -.22 & -.33 & .39 & .48 & & & & & & & & & & \\
\hline 7. Time 1: self-esteem & .17 & .13 & .14 & -.56 & -.37 & -.32 & & & & & & & & & \\
\hline 8. Time 2: self-esteem & .11 & .20 & .15 & -.38 & -.58 & -.34 & .48 & & & & & & & & \\
\hline 9. Time 3: self-esteem & .13 & .18 & .23 & -.33 & -.39 & -.57 & .42 & .48 & & & & & & & \\
\hline 10. Time 1: Grades & .13 & .11 & .08 & -.27 & -.23 & -.15 & .19 & .13 & .13 & & & & & & \\
\hline 11. Time 2: Grades & .15 & .15 & .12 & -.27 & -.35 & -.22 & .17 & .17 & .15 & .58 & & & & & \\
\hline 12. Time 3: Grades & .16 & .14 & .17 & -.25 & -.33 & -.33 & .20 & .18 & .22 & .48 & .61 & & & & \\
\hline 13. Sex & .11 & .09 & .00 & -.04 & -.06 & -.02 & -.03 & -.02 & -.01 & .14 & .19 & .18 & & & \\
\hline 14. Socioeconomic status & -.04 & -.06 & -.01 & .12 & .12 & .05 & -.09 & -.06 & -.04 & -.24 & -.25 & -.20 & -.01 & & \\
\hline 15. Mother's education & .05 & .02 & .04 & -.11 & -.08 & -.11 & .09 & .08 & .06 & .16 & .18 & .17 & .00 & -.18 & \\
\hline 16. Race & .09 & .09 & .05 & -.07 & -.05 & -.02 & -.02 & -.04 & -.05 & .12 & .15 & .12 & .04 & -.33 & .06 \\
\hline
\end{tabular}

Note. Absolute vales of correlations greater than $r=.05$ are significant at the .05 level. 


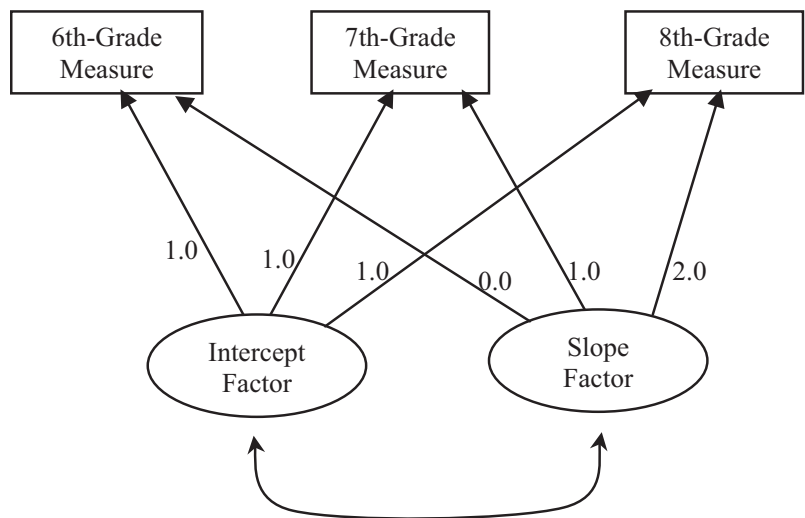

Figure 1. Basic latent growth model with three waves of measurement.

through eighth grades $(t=6,7,8)$. However, the metric for time was centered by subtracting 6 from each time point, thereby creating a time interval of $t=0,1,2$. In addition to ensuring that the intercept represented sixth-grade (initial) status, this rescaling also implied that the slope represented linear rate of change over time. Moreover, all students were assumed to have the same form of growth (linear), but differences might exist in the values of the two latent growth parameters.

The first growth parameter, the intercept, represented the true initial status of the student in sixth grade, and the second growth parameter, the slope, represented the student's true rate of change over time, throughout middle school. For instance, the Level 1 growth model for hours of sleep could be defined as:

$$
Y_{i p}=\pi_{1 p}+\pi_{2 p}\left(t_{i}\right)+\varepsilon_{i p}
$$

where $Y_{i p}$ represented the hours of sleep of student $p$ on occasion $i$ of measurement $(i=1,2,3), t_{i}$ represented the student's grade level (where $t_{i}=0$, $1,2)$, and $\varepsilon_{i p}$ represented the Level 1 measurement errors. The growth parameters were the values of $\pi_{1 p}$ (intercept) and $\pi_{2 p}$ (slope). The intercept parameter $\pi_{1 p}$ represented an estimate of the true average level of hours of sleep in the population when $t_{i}=0$. Students with lower values of this parameter reported lower levels of sleep in sixth grade. The slope parameter $\pi_{2 p}$ represented estimates of the change in true levels of sleep in the population from sixth through eighth grades. Students with higher negative values on this parameter exhibited more rapid decreases in sleep over time. Heterogeneity in growth was captured by the variances of the intercept and slope latent factors (i.e., the variances of $\pi_{1 p}$ and $\pi_{2 p}$, respectively). Similar Level 1 models could be formulated for depressive symptomatology, self-esteem, and grades (for more technical reviews of the aforementioned cross-domain models, refer to Willett \& Keiley, 2000).

\section{Unconditional Models}

We first estimated the unconditional models, one each for the repeated measures of sleep, depressive symptoms, self-esteem, and self-reported grades. This permitted an examination of the average growth trajectories as well as the presence of individual variability across the average growth parameters. It also allowed assessment of the first hypothesis: that as students progress through middle school, they will obtain less sleep and will experience increased levels of depressive symptoms and decreased levels of self-esteem and academic achievement. The roles of sex, SES, race, and mother's education were also examined by regressing these predictors on the intercept and latent growth factors.

Sleep. The unconditional model for hours of sleep converged in five iterations. The overall percentage of missing data reported as part of the FIML procedure was $6.1 \%$. The model fit the data reasonably well, $\chi^{2}(1)=4.01, p<.05$; root mean square error of approximation (RMSEA) $=.037$. In none of the unconditional models were the errors allowed to correlate over time; had this been permitted, better fitting models might have resulted. The average growth trajectory for hours of sleep revealed significant (i.e., different from zero) levels of sleep at sixth grade $(\kappa=3.89, p<.001)$. The estimate of the slope was negative $(\kappa=-0.26)$ and significantly different from zero $(p<.001)$, indicating that the population average slope decreased over the course of middle school at a rate of .26 points (slightly more than $15 \mathrm{~min}$ ) per year. Examination of the variance components of the latent factors showed that both the intercept $(\Phi=.49, p<.001)$ and the slope $(\Phi=.095, p<.001)$ latent factors indicated significant individual differences in both intercept (i.e., sixth grade) levels and declines in hours of sleep over time.

We also wished to explore the effects of participants' demographic characteristics (i.e., sex, mother's education, SES, and race) on sleep; accordingly, both the intercept and slope factors for sleep were regressed on these variables. The model including only the demographic variable of sex had significant paths to both the intercept and the slope. At the beginning of sixth grade, girls reported more sleep than boys $(\gamma=.26, p<.001)$, yet they reported a 
sharper decline over time in hours of sleep than boys $(\gamma=-.12, p<.001)$. This is depicted in Figure 2, which demonstrates the effects of participant sex on sleep. The model with only SES indicated that this variable significantly affected the intercept of sleep $(\gamma=-.14, p<.05)$, such that students with a higher SES obtained more sleep initially; however, this variable did not significantly influence the slope of sleep. Race also significantly affected the intercept of sleep $(\gamma=.28, p<.001)$, such that White students obtained more sleep at the beginning of sixth grade than non-White students; however, race did not significantly influence change in sleep over time. Mother's education was not significant with regard to the intercept or the slope of sleep. Because the model including sex was effective in predicting both the intercept and the slope of sleep, sex was highlighted in the rest of the analyses.

Depressive symptoms. The unconditional model for depressive symptoms converged in four iterations and fit the data reasonably well, $\chi^{2}(1)=12.34, p<.01$; RMSEA $=.07$. The average growth trajectory showed that the initial status of depressive symptoms was significantly different from zero $(\kappa=1.34$, $p<.001)$. A positive slope factor $(\kappa=.023, p<.001)$ indicated increasing levels of depressive symptoms over the course of middle school. Significant variance in both initial status $(\Phi=.06, p<.001)$ and

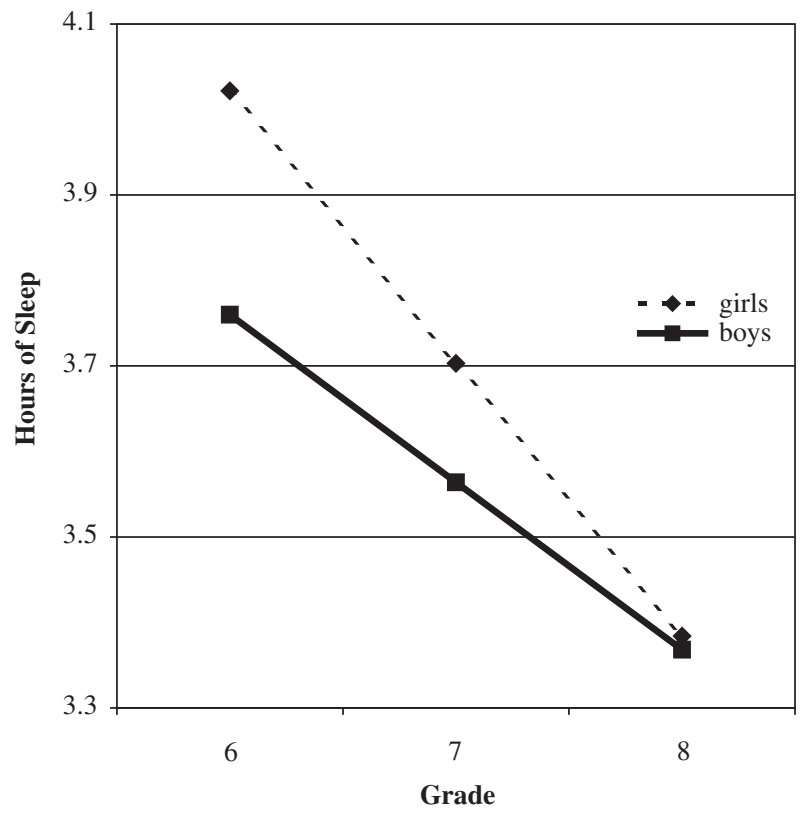

Figure 2. Hours of sleep by sex of adolescent. Girls: sixth grade, $M=4.02$, seventh grade, $M=3.70$, eighth grade, $M=3.38$; Boys: sixth grade, $M=3.76$, seventh grade, $M=3.56$, eighth grade, $M=3.37 .3=7 \mathrm{hr}$ of sleep; $4=8 \mathrm{hr}$ of sleep. slope $(\Phi=.01, p<.001)$ showed that there were significant individual differences in the growth trajectories over time. We also examined the effects of participant sex on depressive symptoms, but there were no significant findings.

Self-esteem. The unconditional model for selfesteem fit the data very well, $\chi^{2}(1)=.98, p<.32$; RMSEA $<.001$. The average growth trajectory revealed significant levels of self-esteem in sixth grade $(\kappa=3.13, p<.001)$. The estimate of the slope was negative $(\kappa=-.08)$ and significantly different from zero $(p<.001)$, indicating decreasing reports of selfesteem over time. Variation in the intercept $(\Phi=.18$, $p<.001)$ and the slope $(\Phi=.02, p<.01)$ revealed the presence of heterogeneity in individual trajectories over time. With regard to the effects of participant sex, at the beginning of sixth grade, boys reported higher self-esteem than girls $(\gamma=-.09, p<.001)$, yet they reported a sharper decline over time in selfesteem than girls $(\gamma=.04, p<.05)$.

Self-reported grades. The unconditional model for self-reported grades fit the data very well, $\chi^{2}(1)=1.06, \quad p<.30 ; \quad$ RMSEA $<.01$. The average growth trajectory for self-reported grades indicated significant levels in sixth grade $(\kappa=4.30, p<.001)$. The estimate of the slope was negative and significantly different from zero $(\kappa=-.15, p<.001)$, indicating that self-reported grades decreased throughout middle school. The significant variation in both the intercept $(\Phi=.86, p<.01)$ and the slope $(\Phi=.20, p<.01)$ indicated that there were significant individual differences in growth trajectories over time. In addition, sex was associated with grades. Girls reported higher grades than boys initially $(\gamma=.22, p<.001)$ and over time $(\gamma=.09, p<.02)$.

\section{Combined Model}

The second aim of the study was to examine the effects of sleep on patterns of well-being over time. Specifically, decreasing hours of sleep were expected to correspond with increased levels of depressive symptoms and decreased levels of self-esteem and self-reported grades. To examine this, we tested a combined model, including all of the unconditional models discussed earlier. This model is displayed in Figure 3, with the appropriate standardized parameter estimates. Once the Level 1, within-person models were specified in the unconditional models, the relationship between these models defined the Level 2, between-person models (which depict interparticipant variability). Thus, the parameter estimates contained in the beta matrix revealed the effects of hours of sleep on the outcomes (depressive 


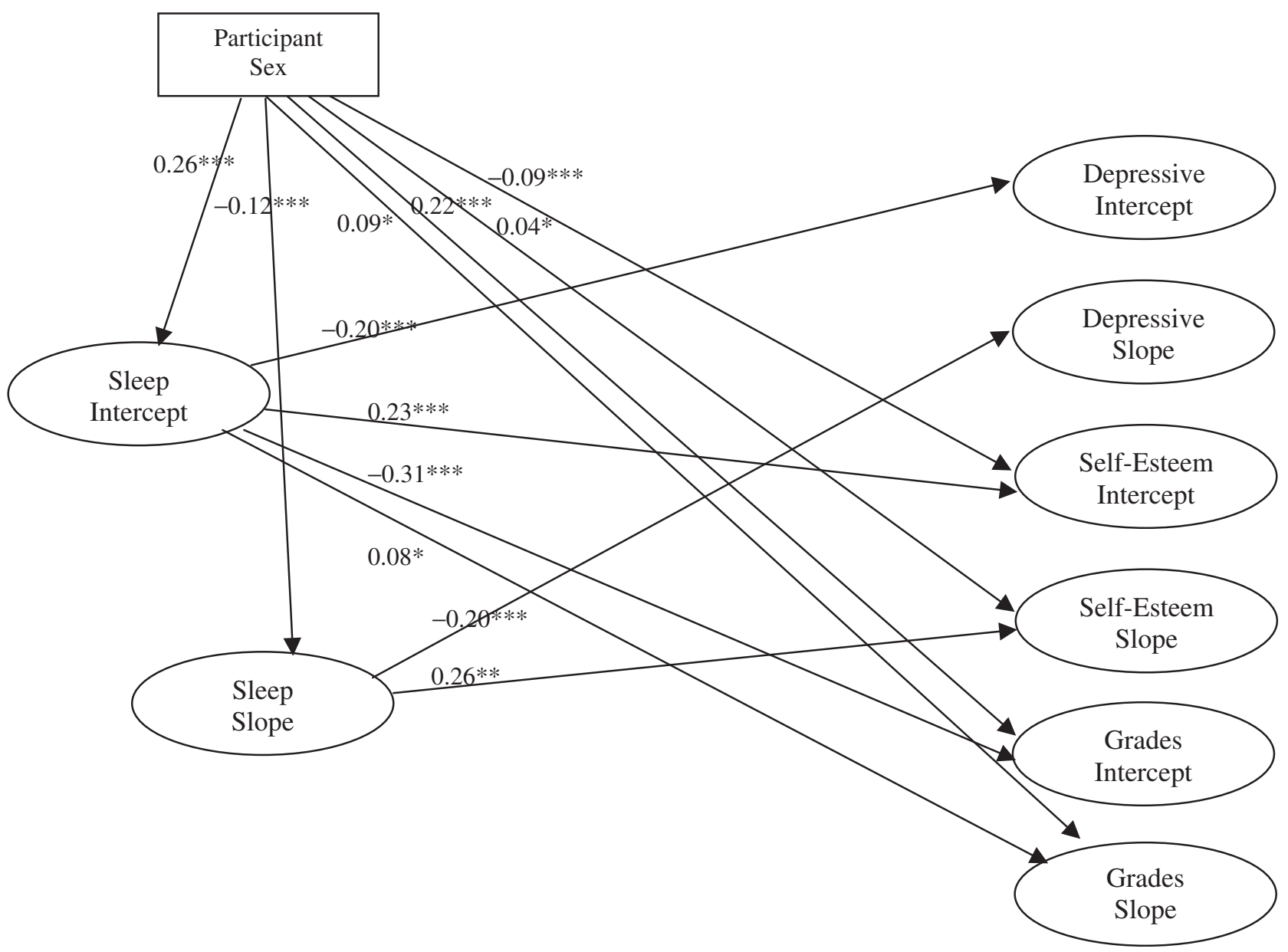

Figure 3. Final combined cross-domain model.

symptoms, self-esteem, and self-reported grades). For example, the latent regression coefficient $\beta_{1}$ indexed the effect of levels of sleep on depressive symptoms in sixth grade, whereas $\beta_{6}$ indexed the impact of the true rate of change in hours of sleep on the rate of change in depressive symptoms. The left side of Figure 3 denotes the individual growth model for hours of sleep, with two latent growth parameters, intercept and slope. The right side of the figure contains the individual growth models for the outcome variables, depressive symptoms, self-esteem, and grades. To assess the influence of initial levels of sleep, the intercepts and slopes of the outcome variables were regressed on the intercept of hours of sleep. To investigate how rates of change in sleep predicted rates of change in the outcome variables, the slopes of the latter were regressed on the slope of hours of sleep. The structural model, which defines the relationship between latent growth models for hours of sleep and the outcome variables, then permits an examination of the relationship of change in one variable on change in the other. This is the primary benefit of a crossdomain approach. In addition, the intercepts and slopes of the variables were regressed on sex (see Figure 3; for the sake of clarity, only significant paths from sex and from the intercept and slope of sleep to the outcome variables are represented).

The combined model fit the data reasonably well, FIML $\chi^{2}(23)=48.93$; RMSEA $=.02$. Sex significantly affected both the intercept and slope of sleep, such that girls initially reported more sleep than boys yet reported a sharper decline over time. Sex also influenced the intercepts and slopes of self-esteem and grades, such that boys initially reported higher self-esteem than girls yet reported a sharper decline over time, and girls reported higher grades than boys both initially and over time. The intercept of sleep significantly influenced the intercepts of depressive symptoms, self-esteem, and grades, such that students who initially reported higher levels of sleep also tended to report lower levels of depressive 
symptoms $(\beta=-.20, p<.001)$, higher levels of selfesteem $(\beta=.23, p<.001)$, and higher grades $(\beta=.31$, $p<.001)$. The slope of sleep (i.e., its rate of change) significantly affected the slopes of depressive symptoms and self-esteem, such that students who reported more sleep over time also reported fewer depressive symptoms $(\beta=-.20, p<.001)$ and higher self-esteem $(\beta=.27, p<.01)$. However, the effect on grades was not significant. Other than this last result, all of the other findings accord with the hypotheses stated earlier.

\section{Alternative Model}

It could be argued that the demonstrated effects between level of sleep and academic and psychosocial outcomes were in fact working solely in the opposite direction; that is, rather than hours of sleep predicting depressive symptoms, self-esteem, and self-reported grades, the psychosocial and academic variables may have been responsible for changes in sleep patterns. After all, one of the symptoms of depression is loss of sleep. To counter such arguments and to demonstrate that sleep was indeed a strong predictor of psychosocial and academic variables, it was necessary to test this alternative model. In this model, the variable hours of sleep was considered an outcome variable, and the three other primary variables (depressive symptoms, self-esteem, and self-reported grades) were entered as predictors. Sex was included in the model as defined earlier. We could thus make qualitative comparisons of model effects to gain some degree of insight regarding the ordering of effects from sleep to depressive symptoms, self-esteem, and grades. The alternative model was estimated using the FIML procedure. It fit the data reasonably well, $\chi^{2}(23)=47.34$; RMSEA $=.02$. However, except for the influence of the intercept of depressive symptoms on the intercept of sleep $(\gamma=-1.75, p<.001)$, none of the remaining pathways relating depressive symptoms, self-esteem, and self-reported grades to the intercept and slope of hours of sleep were significant. In the original model, initial levels of sleep significantly predicted initial levels of depressive symptoms, self-esteem, and self-reported grades. In the alternative model, although the first pathway was valid, the latter two were not, (self-esteem: $\gamma=$ $-.27, p>.05$; grades: $\gamma=.03, p>.05$ ). In addition, in the original model, the slope of hours of sleep significantly predicted the slopes of depressive symptoms and self-esteem, yet in this analysis neither the slope of depressive symptoms $(\gamma=-1.74, p>.05)$, nor the slope of self-esteem $(\gamma=.15, p>.05)$, nor the slope of grades $(\gamma=-.06, p>.05)$ significantly predicted the slope of sleep. Hence, this suggests that psychosocial trajectories were indeed influenced by trajectories of sleep, rather than vice versa.

\section{Discussion}

The main goal of this study was to explore adolescent sleep patterns over the course of middle school. Specifically, we hypothesized that adolescents would obtain decreasing amounts of sleep and that this chronic insufficient sleep would predict changes in depressive symptoms, self-esteem, and grades; those who obtained less sleep would experience a rise in depressive symptoms and decreases in self-esteem and grades. Using an idiographic approach, we examined interindividual pathways of change in these variables. Consistent with previous research, we found that hours of sleep did indeed decrease during the middle school years for both males and females, and especially for the latter group. Students who experienced lower levels of sleep at the beginning of sixth grade also exhibited lower selfesteem and grades and higher levels of depressive symptoms at that point. By the same token, students who obtained less sleep over time experienced heightened depressive symptoms and decreased self-esteem. Although grades dropped over time, the combined model did not produce a significant result for the effect of the slope of sleep on the slope of grades. Except for this last result, our findings corresponded with the hypotheses stated earlier.

Viewed together, these findings add to previous studies that have revealed sleep loss during adolescence and build on this area of research by illuminating some of the ways in which this insufficient sleep can be detrimental. In addition, the findings highlight the importance of tracking measures of sleep and psychosocial and academic outcomes as they change over time. Individual growth curves demonstrated the dynamic pattern of relationships among the variables. Indeed, the primary strength of this study lies in its methodology. Cross-domain modeling allows greater accuracy in evaluating adolescence, a period of transition and flux. Although it remains impossible to designate direct causal pathways with absolute certainty, the posthoc analysis indicated that, barring a single bidirectional pathway (between the intercepts of depressive symptoms and sleep), the direction of influence between sleep and the outcome variables was largely unidirectional. This challenges the argument that the outcome variables were, in fact, responsible for the changes in sleep. Sleep clearly 
played a significant role in predicting depressive symptoms and self-esteem during adolescence.

Another discovery involved the role of sex as a predictor. At the beginning of middle school, girls obtained more sleep than boys. One explanation for this difference may be the earlier onset of puberty for girls, which corresponds with a heightened need for sleep (Laberge et al., 2001). It is interesting, however, that although levels of sleep dropped for both groups over time, this decline occurred at a steeper rate for girls. This is consistent with research that has shown that, although boys and girls have similar weekday bedtimes, boys tend to wake up later than girls on weekdays (Lee et al., 1999). Researchers have suggested that girls' earlier wakeup times may be due to more substantial morning grooming routines or a greater load of household chores, or both (Giannotti \& Cortesi, 2002; Lee et al., 1999). These differences are a disturbing reminder of how early gender role expectations may come into play. Future studies that track the time and rationale for bed and wake-up times would help to further illuminate these trends. It would also be important to explore sex differences in the quality of adolescent sleep over time. Reports are conflicting in this regard, with some studies reporting greater sleep disturbance for girls (e.g., Giannotti \& Cortesi, 2002) and others reporting no sex differences in sleep quality (e.g., Lee et al., 1999; Liu \& Zhou, 2002).

Sex also had an effect on some of the outcome variables. Boys initially reported higher levels of self-esteem than girls, a result that is generally supported in the literature (Bolognini, Plancherel, Bettschart, \& Halfon, 1996; Eccles, Barber, Jozefowicz, Malenchuk, \& Vida, 2002; Kling et al., 1999). Although drops in self-esteem are expected for both boys and girls during adolescence (Robins, Trzesniewski, Tracy, Gosling, \& Potter, 2002), boys' selfesteem dropped more precipitately than girls' over time. Girls reported higher grades initially and over time. This finding corresponds with data showing that girls typically outperform boys academically from childhood through adolescence (Pomerantz, Altermatt, \& Saxon, 2002).

Other contextual features (e.g., race and SES), which were not fully explored in this relatively homogeneous sample, may also have influenced the results. We found, for example, that higher SES students (i.e., students who were not eligible for a free or reduced price lunch) obtained more sleep at the beginning of sixth grade. We also found that White students obtained more sleep at the beginning of sixth grade than non-White students. Future research should use more culturally and economic- ally diverse samples and focus on the potential moderating effects of participant race and SES on sleep patterns (e.g., through cramped and noisy living space, and employment patterns; Durrence, Lichstein, Taylor, Bush, \& Reidel, 2001).

Our study is limited by the reliance on singleitem, self-report measures of grades and sleep. With respect to grades, it is noteworthy that other researchers have used a similar question to assess school performance (Dornbusch, Ritter, Leiderman, Roberts, \& Fraleigh, 1987; Wolfson \& Carskadon, 1998), and self-reported grades have been found to offer a close approximation of transcripts (Dornbusch et al., 1987). Indeed, in the current study, selfreported grades in seventh and eighth grades were highly positively correlated with teacher-reported grades in seventh grade $(r=.62, p<.01)$ and eighth grade $(r=.61, p<.01)$. Nonetheless, actual transcripts and other indexes of academic progress would have strengthened our claims.

Along similar lines, our single-item assessment of sleep was not ideal, and future work should provide a more thorough index of both the quality and quantity of adolescents' sleep patterns. Previous researchers have delved into a broad range of sleep characteristics (e.g., disturbances, schedule, daytime fatigue), and such information would have enriched this study. With respect to quantity, narrower increments would have provided a more nuanced index, particularly because sleep restrictions of a half hour have been shown to affect functioning (Sadeh, Gruber, \& Raviv, 2003). Physiological indexes, employing special equipment and sleep laboratory data, would have provided more definitive data regarding sleep-wake cycles (Carksadon et al., 1997; Carskadon et al., 1998). There are trade-offs, however, between the precision of smaller physiological studies and the ecological validity of larger self-report studies, and many researchers have relied on the latter (e.g., Roberts et al., 2001; Wolfson \& Carskadon, 1998). Fortunately, previous work has established strong associations between physiological and self-reported measures of sleep (Roberts et al., 2001; Wolfson \& Carskadon, 1998). Finally, our method of assessing sleep quantity is generally in line with the self-report measures that have been employed in previous studies. Although many (e.g., Lee et al., 1999; Wolfson \& Carskadon, 1998) request bedtime and wake-up time hours as opposed to a report of students' hours of sleep on a typical school night, both approaches yield essentially the same information.

Despite these limitations, this study has important implications for research and policy. Elevated levels of depression and drops in self-esteem are 
seen as inevitable hallmarks of adolescence. Yet these results suggest that such changes are partially linked to a variable that is largely under individual, parental, and even school control. Thus, any attempts to improve the quality of life for adolescents and reduce their risk to a range of negative health, academic, and emotional outcomes should consider the importance of a good night's sleep. Teachers could devote attention to the topic in health and physical education classes, and parents could encourage earlier bedtimes, especially on school nights. Where possible, efforts should be made to encourage lighter homework loads and later school start times, so that adolescents can go to bed and wake up at times that are more suited to their bodily rhythms. Continued longitudinal research designed to better understand the effects of adolescent sleep loss on a broad array of developmental outcomes should go hand in hand with attempts to counteract its detrimental effects.

\section{References}

Allgoewer, A., Wardle, J., \& Steptoe, A. (2001). Depressive symptoms, social support, and personal health behaviors in young men and women. Health Psychology, 20, $223-227$

Andrade, M., \& Menna-Barreto, L. (2002). Sleep patterns of high school students living in Sao Paulo, Brazil. In M. A. Carksadon (Ed.), Adolescent sleep patterns: Biological, social, and psychological influences (pp. 118-131). New York: Cambridge University Press.

Bolognini, M., Plancherel, B., Bettschart, W., \& Halfon, O. (1996). Self-esteem and mental health in early adolescence: Development and gender differences. Journal of Adolescence, 19, 233-245.

Carskadon, M. A. (1990a). Adolescent sleepiness: Increased risk in a high-risk population. Alcohol, Drugs, and Driving, 5, 317-328.

Carskadon, M. A. (1990b). Patterns of sleep and sleepiness in adolescents. Pediatrician, 17, 5-12.

Carskadon, M. A. (1999). When worlds collide: Adolescent need for sleep versus societal demands. Phi Delta Kappan, 80, 348-353.

Carskadon, M. A. (2002). Factors influencing sleep patterns of adolescents. In M. A. Carksadon (Ed.), Adolescent sleep patterns: Biological, social, and psychological influences (pp. 4-26). New York: Cambridge University Press.

Carskadon, M. A., Acebo, C., Richardson, G. S., Tate, B. A., \& Seifer, R. (1997). An approach to studying circadian rhythms of adolescent humans. Journal of Biological Rhythms, 12, 278-289.

Carskadon, M. A., \& Dement, W. C. (1987). Sleepiness in the normal adolescent. In C. Guilleminault (Ed.), Sleep and its disorders in children (pp. 53-67). New York: Raven Press.
Carskadon, M. A., Harvey, K., Duke, P., Anders, T. F., \& Dement, W. C. (1980). Pubertal changes in daytime sleepiness. Sleep, 2, 453-460.

Carskadon, M. A., Labyak, S. E., Acebo, C., \& Seifer, R. (1999). Intrinsic circadian period of adolescent humans measured in conditions of forced desynchrony. Neuroscience Letters, 260, 129-132.

Carskadon, M. A., Vieira, C., \& Acebo, C. (1993). Association between puberty and delayed phase preference. Sleep, 16, 258-262.

Carskadon, M. A., Wolfson, A. R., Acebo, C., Tzischinsky, O., \& Seifer, R. (1998). Adolescent sleep patterns, circadian timing, and sleepiness at a transition to early school days. Sleep, 21, 871-881.

Choquet, M., Kovess, V., \& Poutignat, N. (1993). Suicidal thoughts among adolescents: An intercultural approach. Adolescence, 28(111), 649-659.

Dahl, R. E., \& Carskadon, M. A. (1995). Sleep and its disorders in adolescence. In R. Ferber \& M. H. Kryger (Eds.), Principles and practice of sleep medicine in the child (pp. 19-27). Philadelphia: Saunders.

Dahl, R. E., \& Lewin, D. S. (2002). Pathways to adolescent health: Sleep regulation and behavior. Journal of Adolescent Health, 31, 175-184.

Dornbusch, S. M. (2002). Sleep and adolescence: A social psychologist's perspective. In M. A. Carskadon (Ed.), Adolescent sleep patterns: Biological, social, and psychological influences (pp. 1-3). New York: Cambridge University Press.

Dornbusch, S. M., Ritter, P. L., Leiderman, P. H., Roberts, D. F., \& Fraleigh, M. J. (1987). The relation of parenting style to adolescent school performance. Child Development, 58, 1244-1258.

DuBois, D. L., Bull, C. A., Sherman, M. D., \& Roberts, M. (1998). Self-esteem and adjustment in early adolescence: A social-contextual perspective. Journal of Youth and Adolescence, 27, 557-583.

DuBois, D. L., \& Felner, R. D. (1991). [Reliability and validity data for the self-esteem questionnaire]. Unpublished raw data.

DuBois, D. L., Felner, R. D., Brand, S., \& George, G. R. (1999). Profiles of self-esteem in early adolescence: Identification and investigation of adaptive correlates. American Journal of Community Psychology, 27, $899-932$.

DuBois, D. L., Felner, R. D., Brand, S., Phillips, R. S. C., \& Lease, A. M. (1996). Early adolescent self-esteem: A developmental-ecological framework and assessment strategy. Journal of Research on Adolescence, 6, 543-579.

Durrence, H. H., Lichstein, K. L., Taylor, D. J., Bush, A. J., \& Riedel, B. W. (2001, June). The role of ethnicity on sleep and health. Chicago: Poster presented at the meeting of the Association of Professional Sleep Societies.

duToit, M., \& duToit, S. (2001). Interactive LISREL: User's guide. Chicago: Scientific Software International.

Eccles, J., Barber, B., Jozefowicz, D., Malenchuk, O., \& Vida, M. (2002). Self-evaluations of competence, task values and self-esteem. In N. G. Johnson, M. C. Roberts, \& J. Worell (Eds.), Beyond appearance: A new look at 
adolescent girls (pp. 53-84). Washington, DC: American Psychological Association.

Furstenberg, F. F. Jr., Cook, T. D., Eccles, J., Elder, G. H. Jr., \& Sameroff, A. (1999). Managing to make it: Urban families and adolescent success. Chicago: University of Chicago Press.

Giannotti, F., \& Cortesi, F. (2002). Sleep patterns and daytime function in adolescence: An epidemiological survey of an Italian high school student sample. In M. A. Carksadon (Ed.), Adolescent sleep patterns: Biological, social, and psychological influences (pp. 132-147). New York: Cambridge University Press.

Giannotti, F., Cortesi, F., Sebastiani, T., \& Ottaviano, S. (2002). Circadian preference, sleep and daytime behaviour in adolescence. Journal of Sleep Research, 11, 191-199.

Hall, A. S., \& Parsons, J. (2001). Internet addiction: College student case study using best practices in cognitive behavior therapy. Journal of Mental Health Counseling, 23, $312-327$.

Hankin, B. L., \& Abramson, L. Y. (2001). Development of gender differences in depression: An elaborated cognitive vulnerability-transactional stress theory. Psychological Bulletin, 127, 773-796.

Jöreskog, K., \& Sörbom, D. (2001). LISREL 8.30 [Computer software]. Chicago: Scientific Software International.

Kandell, J. J. (1998). Internet addiction on campus: The vulnerability of college students. CyberPsychology $\mathcal{E}$ Behavior, 1, 11-17.

Kazdin, A. E. (1989). Identifying depression in children: A comparison of alternative selection criteria. Journal of Abnormal Child Psychology, 17, 437-454.

Kling, K. C., Hyde, J. S., Showers, C. J., \& Buswell, B. N. (1999). Gender differences in self-esteem: A metaanalysis. Psychological Bulletin, 125, 470-500.

Kovacs, M. (1980). Affective disorders in children and adolescents. American Psychologist, 44, 209-215.

Kovacs, M., \& Devlin, B. (1998). Internalizing disorders in childhood. Journal of Child Psychology and Psychiatry, 39, $47-63$.

Laberge, L., Petit, D., Simard, C., Vitaro, F., Tremblay, R. E., \& Montplaisir, J. (2001). Development of sleep patterns in early adolescence. Journal of Sleep Research, 10, 59-67.

Lee, K. A., McEnany, G., \& Weekes, D. (1999). Gender differences in sleep patterns for early adolescents. Journal of Adolescent Health, 26, 16-20.

Leotta, C., Carskadon, M. A., Acebo, C., Seifer, R., \& Quinn, B. (1997). Effects of acute sleep restriction on affective response in adolescents: Preliminary results. Sleep Research, 26, 201.

Liu, X., \& Zhou, H. (2002). Sleep duration, insomnia, and behavioral problems among Chinese adolescents. Psychiatry Research, 111, 75-85.

Park, Y. M., Matsumoto, K., Seo, Y. J., Kang, M. J., \& Nagashima, H. (2002). Changes of sleep or waking habits by age and sex in Japanese. Perceptual and Motor Skills, 94, 1199-1213.

Park, Y. M., Matsumoto, K., Shinkoda, H., Nagashima, H., Kang, M. J., \& Seo, Y. J. (2001). Age and gender difference in habitual sleep-wake rhythm. Psychiatry and Clinical Neurosciences, 55, 201-202.
Pomerantz, E. M., Altermatt, E. R., \& Saxon, J. L. (2002). Making the grade but feeling distressed: Gender differences in academic performance and internal distress. Journal of Educational Psychology, 94, 396-404.

Randazzo, A. C., Muehlbach, M. J., Schweitzer, P. K., \& Walsh, J. K. (1998). Cognitive function following acute sleep restriction in children ages 10-14. Sleep, 21, $861-868$.

Roberts, R. E., Roberts, C. R., \& Chen, I. G. (2001). Functioning of adolescents with symptoms of disturbed sleep. Journal of Youth and Adolescence, 30, 1-18.

Robins, R. W., Trzesniewski, K. H., Tracy, J. L., Gosling, S. D., \& Potter, J. (2002). Global self-esteem across the life span. Psychology and Aging, 17, 423-434.

Sadeh, A., Gruber, R., \& Raviv, A. (2003). The effects of sleep restriction and extension on school-age children: What a difference an hour makes. Child Development, 74, $444-455$.

Sadeh, A., Raviv, A., \& Gruber, R. (2000). Sleep patterns and sleep disruptions in school-age children. Developmental Psychology, 36, 291-301.

Saylor, C. F., Finch, A. J., Spirito, A., \& Bennett, B. (1984). The Children's Depressive Inventory: A systematic evaluation of psychometric properties. Journal of Consulting and Clinical Psychology, 52, 955-967.

Sitarenios, G., \& Kovacs, M. (1999). Use of the Children's Depression Inventory. In M. E. Maruish (Ed.), The use of psychological testing for treatment planning and outcomes assessment (2nd ed., pp. 267-298). Mahwah, NJ: Erlbaum.

Tvenge, J. M., \& Nolen-Hoeksema, S. (2002). Age, gender, race, socioeconomic status, and birth cohort differences on the Children's Depressive Inventory: A metaanalysis. Journal of Abnormal Psychology, 111, 578-588.

Wahlstrom, K. (1999). The prickly politics of school starting times. Phi Delta Kappan, 80, 344-347.

Wahlstrom, K. (2002). Accommodating the sleep patterns of adolescents within current educational structures: An uncharted path. In M. A. Carksadon (Ed.), Adolescent sleep patterns: Biological, social, and psychological influences (pp. 172-197). New York: Cambridge University Press.

Willett, J. B., \& Keiley, M. K. (2000). Using covariance structure analysis to model change over time. In H. E. A. Tinsley \& S. Brown (Eds.), Handbook of applied multivariate statistics and mathematical modeling: A comprehensive guide for applied researchers in the biological sciences, social sciences, and humanities (pp. 665-694). San Diego, CA: Academic Press.

Wolfson, A. R. (2002). Bridging the gap between research and practice: What will adolescents' sleep-wake patterns look like in the 21st century? In M. A. Carksadon (Ed.), Adolescent sleep patterns: Biological, social, and psychological influences (pp. 198-219). New York: Cambridge University Press.

Wolfson, A. R., \& Carskadon, M. A. (1998). Sleep schedules and daytime functioning in adolescents. Child Development, 69, 875-887.

Young, K. (1998). Internet addiction: The emergence of a new clinical disorder. CyberPsychology and Behavior, 1, $237-244$. 